\title{
Retraction of: Tumor Protein D52-Like 2 Contributes to Proliferation of Breast Cancer Cells; 10.1089/cbr.2014.1723
}

Cancer Biotherapy and Radiopharmaceuticals (CBR) is officially retracting the published article by Mei Yang, Xueyao Wang, Jiaoyuan Jia, Hongwen Gao, Peng Chen, Xianliang Sha, and Shan Wu entitled, “Tumor Protein D52-Like 2 Contributes to Proliferation of Breast Cancer Cells,'” Cancer Biother Radiopharm February 2015, 30(1): 1-7. doi:10.1089/ cbr.2014.1723.

The editors of CBR received a letter from an investigator raising concerns regarding this published paper. Upon examining the 5'-GCGG.... shRNA sequence described in the article, the investigator discovered that the lentiviral sequence cited to serve as a "non-silencing" control in fact shows homology to the human TPD52L2 gene (as per a Blastn search conducted by the investigator). The investigator claimed that the article therefore reported the results of knocking down the TPD52L2 gene as both the gene of interest and the supposed negative control.

The investigator's concern was such that since the article employed an incorrect control lentiviral construct, the experimental results reported must be invalid. The editors of the CBR contacted the authors of the published paper who confirmed that the sequence was wrong which may have occurred due to a "copy paste error," and agreed that the report could be misleading and agreed to retract the paper from the literature. This decision is also based on our published understanding of the incorrect use of nucleotide sequence reagents by this publication.

It is important to note that an earlier e-pub version of this retraction notice was posted with unintended errors and have been corrected within this official retraction notice. A list of the affected text appears below.

Cancer Biotherapy and Radiopharmaceuticals is dedicated to upholding the strictest standards of peer review and the scientific record and therefore is retracting this article.

\section{List of corrected text from e-published versions of this retraction:}

Original retraction text: ...the investigator discovered that the lentiviral sequence cited to target TPD52L2 actually does not correspond to any TPD52L2 sequence, and does not show homology to any sequence in the non-redundant nucleotide database (as per a Blastn search conducted by the investigator).

- CORRECTED text: ...the investigator discovered that the lentiviral sequence cited to serve as a "non-silencing", control in fact shows homology to the human TPD52L2 gene (as per a Blastn search conducted by the investigator).

Original retraction text: The investigator claimed that the article reported using the $5^{\prime}$-GCGG... shRNA as a non-targeting control, but the alternative "TPD52L2-targeting" 5'-CCGG... shRNA does not target the TPD52L2 gene as described, or any other sequence in the non-redundant nucleotide database.

- CORRECTED text: The investigator claimed that the article therefore reported the results of knocking down the TPD52L2 gene as both the gene of interest and the supposed negative control.

Original retraction text: The investigator's concern was such that since the article did not employ a lentiviral construct targeting the TPD52L2 gene, the experimental results reported must be invalid.

- CORRECTED text: The investigator's concern was such that since the article employed an incorrect control lentiviral construct, the experimental results reported must be invalid. 